\title{
The Influence of Obesity and Metabolic Health on Vascular Health
}

\section{Eun-Jung Rhee}

Department of Endocrinology and Metabolism, Kangbuk Samsung Hospital, Sungkyunkwan University School of Medicine, Seoul, Korea

The prevalence of obesity is rapidly increasing worldwide. Obesity should not be understood only as the accumulation of fat in the body, but instead as a phenomenon that exerts different effects on our health according to the place of fat deposition and its stability. Obesity is the starting point of most metabolic diseases, such as diabetes, hypertension, metabolic syndrome, sleep apnea, and eventually cardiovascular disease. There are different kinds of obesity, ranging from simple obesity to sarcopenic obesity. The main purpose of intervening to address obesity is to decrease the ultimate consequence of obesity - namely, cardiovascular disease. The main mechanism through which obesity, especially abdominal obesity, increases cardiovascular risk is the obesity-induced derangement of metabolic health, leading to the development of metabolic diseases such as diabetes, non-alcoholic fatty liver disease, and metabolic syndrome, which are the main initiators of vascular damage. In this review, I discuss the influence of various types of obesity on the risk of metabolic diseases, and how these diseases increase cardiovascular disease risk.

Keywords: Atherosclerosis; Cardiovascular diseases; Diabetes mellitus; Metabolism; Obesity; Vascular calcification

The Namgok Award is the highest scientific award of the Korean Endocrine Society, and is given to honor an individual who has made excellent contributions to progress in the field of endocrinology and metabolism. The Namgok Award is named after the pen name of Professor Hun Ki Min, who founded the Korean Endocrine Society in 1982. Professor Eun-Jung Rhee received the Namgok Award at the 9th Seoul International Congress of Endocrinology and Metabolism of the Korean Endocrine Society in October 2021.

Received: 25 January 2022, Revised: 30 January 2022,

Accepted: 8 February 2022

Corresponding author: Eun-Jung Rhee

Department of Endocrinology and Metabolism, Kangbuk Samsung Hospital, Sungkyunkwan University School of Medicine, 29 Saemunan-ro, Jongno-gu, Seoul 03181, Korea

Tel: +82-2-2001-2485, Fax: +82-2-2001-2049, E-mail: hongsiri@hanmail.net

\section{INTRODUCTION}

Obesity is renowned for its tendency to be accompanied by comorbid diseases, such as diabetes, atherosclerosis, metabolic syndrome (MS), and sleep apnea [1-3]. The prevalence of obesity in adults older than 30 has reached $30 \%$ worldwide, and this high rate of obesity is particularly pronounced in Asians, due to rapid changes in dietary patterns and a sedentary lifestyle [4].

Abdominal obesity is known to be a major risk factor for diabetes and cardiovascular disease (CVD) [5]. This increased risk for metabolic diseases results from insulin resistance, which

\section{Copyright $(92022$ Korean Endocrine Society}

This is an Open Access article distributed under the terms of the Creative Commons Attribution Non-Commercial License (https://creativecommons.org/ licenses/by-nc/4.0/) which permits unrestricted non-commercial use, distribution, and reproduction in any medium, provided the original work is properly cited. 
visceral fat causes differently from subcutaneous fat accumulation. The presence of abdominal obesity indicates that ectopic fat infiltration has started in a person's body, and this is not only confined to the abdomen. Ectopic fat accumulation is known to be caused by insulin resistance; more specifically, our body resists the role of insulin in promoting the storage of excess energy as triglycerides in adipose tissue, and excess fat therefore accumulates in places other than adipose tissue, such as the liver, muscle, abdomen, and perivascular area [6,7]. These ectopically accumulated fat deposits derange organ function, causing insulin resistance in the target organ. In turn, insulin resistance causes metabolic diseases such as non-alcoholic fatty liver disease (NAFLD), MS, and type 2 diabetes.

The Kangbuk Samsung Health Study (KSHS) is one of the largest health screening cohorts currently ongoing in Koreans, supporting prospective and retrospective research, and it screens more than 150,000 subjects every year. As a clinician and researcher working at Kangbuk Samsung Hospital, Sungkyunkwan University School of Medicine, the author has had the opportunity to have full access to the KSHS dataset.

The Korean National Health Insurance Service (NHIS) system is a mandatory health insurance program that covers $97.1 \%$ of the Korean population [8]. In Korea, the NHIS is the single insurer, managed by the government. The NHIS includes an eligibility database, a medical treatment claims database (based on medical bills that were claimed by medical service providers for their medical expenses), a health examination database (with results of general health examinations and questionnaires on lifestyle and behavior), a medical care institution database (types of medical care institutions, location, equipment, and number of physicians), and a death register.

This review discusses the clinical implications, based on upto-date knowledge, of the influence of obesity and metabolic health on vascular health, with a primary focus on the results of analyses of KSHS and NHIS data drawn from the author's own work.

\section{TRENDS IN THE PREVALENCE OF OBESITY AND DIABETES IN KOREA AND THEIR CONSEQUENCES}

In recent decades, the prevalence of risk factors for CVD, such as diabetes, hypertension, obesity, and dyslipidemia, has been steadily increasing in Koreans. According to the Obesity Fact Sheet released by Korean Society for the Study of Obesity in 2020 , based on NHIS data in 2018, the prevalence of obesity in
Korea was 32.5\% [4]. According to the Diabetes Fact Sheet released by the Korean Diabetes Association (KDA) in 2020, the prevalence of diabetes was $13.8 \%$ in Koreans older than 30 years, based on 2018 data from the Korean National Health and Nutrition Examination Survey [9]. In addition, the prevalence of hypertension in the total population of Korea in 2018 was $28.3 \%$ according to the Hypertension Fact Sheet produced by the Korean Society of Hypertension [10]. Lastly, the prevalence of dyslipidemia in 2018 was $40.5 \%$ according to the Dyslipidemia Fact Sheet published by the Korean Society of Lipid and Atherosclerosis [11]. The prevalence of all these risk factors has steadily increased over the last decade according to these fact sheets.

Not only is the prevalence of these risk factors rising, but their incidence is also increasing. According to the "Diabetes \& Complications in Korea" report released by the KDA, during the period of 2006 to 2015, the prevalence of macrovascular complications - ischemic heart disease, stroke, and myocardial infarction - all tended to decline [12]. However, rising trends were seen in the rates of heart failure (HF) and peripheral artery disease, as well as diabetic retinopathy and diabetic nephropathy among Korean patients with diabetes. Diabetic nephropathy was the most common cause of end-stage renal disease (ESRD), and the prevalence of ESRD also increased.

In conclusion, the prevalence of risk factors for CVDs has been gradually rising for the last decade despite the efforts of physicians and healthcare providers in these fields. A particularly ominous trend is the increase in microvascular complications, such as ESRD and loss of vision.

\section{METABOLIC SYNDROME, METABOLICALLY HEALTHY OBESITY, AND CARDIOVASCULAR RISK}

MS is defined as a cluster of metabolic risk factors, such as abdominal obesity, hyperglycemia, hypertension, and dyslipidemia [13]. Although there have been disagreements regarding the definition of MS, the currently accepted definition is the presence of two or more of five risk factors, as suggested by harmonized consensus of multiple associations [14]. MS is a syndrome in which insulin resistance, which is caused by abdominal obesity, induces various CVD risk factors and further causes diabetes and CVD.

After the existence of MS was proposed by Prof. Reaven in 1988 as "Syndrome X," many associations attempted to define MS [15]. A debate ensued regarding the application of the same waist circumference (WC) cutoff for the definition of MS in 
Asians as in Caucasians. Our group argued for the first time in the literature that different WC cutoffs should be applied to define MS in Asians and Caucasians, based on results showing a significantly lower prevalence of MS when different WC and body mass index values were applied in these ethnic groups [16]. Based on our suggestions, different WC cutoffs have been recommended for the definition of MS in Asians and Caucasians.

Some debates took place regarding the components of the MS definition, and in particular whether diabetes and hypertension should be counted as components of MS [17,18]. Our group analyzed the differences that would arise depending on whether the definition of MS included or excluded patients with diabetes or hypertension [19]. In 159,971 Korean participants in KSHS without a previous history of CVD from 2002 to 2009 with a median follow-up of 3.7 years, the mortality rate was assessed within the same period. We observed higher risks for all-cause and CVD mortality in subjects with MS than in those who did not have MS. However, these increased risks were attenuated after the exclusion of subjects with diabetes or hypertension, suggesting that inclusion of these metabolic diseases accounts for the increased CVD mortality attributable to MS, challenging the definition of MS used at that time.

In the early 2010s, a slightly different concept from MS, the concept of "metabolically healthy obesity" (MHO), was introduced [20]. MHO refers to the concept that there is a type of "healthy" obesity. People with MHO are obese, but have less abdominal obesity, higher muscle mass, a higher level of mitochondrial function, and better metabolic flexibility than metabolically unhealthy (MUH) obese people.

Our group designed a study to analyze the cardiovascular status of people with MHO using a subclinical atherosclerosis marker, the coronary artery calcium score (CACS). The CACS is known to be a global indicator of a patient's coronary heart disease (CHD) risk by virtue of its strong association with the total coronary atherosclerotic disease burden [21]. Many cohort studies, for example the Multi-Ethnic Study of Atherosclerosis (MESA) study, have shown that the baseline CACS predicts future CHD events very well, suggesting that the CACS can serve as a predictive marker for CHD risk [22,23].

We analyzed the association between metabolic health and the CACS in 24,063 participants in the KSHS and found that the CACS was significantly higher in MUH people than in metabolically healthy people regardless of obesity, suggesting the existence of MHO [24]. We analyzed the risk of developing diabetes in people with $\mathrm{MHO}$, and found that people with $\mathrm{MHO}$ had a lower risk of diabetes than MUH obese people [25]. Al- though both of our studies demonstrated the existence of MHO as a distinct phenotype, many subsequent studies have debated the existence of $\mathrm{MHO}$, arguing that it is highly dependent on the definition [26].

\section{INSULIN RESISTANCE, HYPERGLYCEMIA, AND SUBCLINICAL ATHEROSCLEROSIS}

Hyperglycemia is known to increase the risk for atherosclerosis and mortality through increased levels of advanced glycation end-products and increased oxidative stress [27]. In 241,299 KSHS participants between 2005 and 2012, the risk of death from all causes, CVD, and cancer was calculated based on the baseline glycemic status (normoglycemia, prediabetes, and diabetes) and glycated hemoglobin (HbA1c) levels [28]. The risks of all-cause and cancer mortality were significantly higher in patients with baseline diabetes than in those with normoglycemia. In subjects not taking anti-diabetic medications, both high and low HbA1c levels were associated with an elevated risk for all-cause mortality.

The presence of diabetes or hyperglycemia increases the risk for coronary artery calcification (CAC) development. In 2,076 subjects without diabetes who had a CACS of 0 at baseline, the risk for CAC development was assessed according to baseline glycemic status [29]. Over 4 years, the subjects who developed diabetes showed the highest proportion of subjects with incident $\mathrm{CAC}$ among the three groups. Insulin resistance was associated with a significantly increased risk for incident CAC, both in subjects who progressed from normal to impaired fasting glycemia and in subjects who developed diabetes. However, subjects without insulin resistance at baseline showed no significantly increased risk for incident $\mathrm{CAC}$, even in those who developed diabetes, suggesting that the increased risk of incident CAC in relation to diabetes development was pronounced only in subjects with insulin resistance at baseline.

From the findings of this study, we could conclude that hyperglycemia and insulin resistance showed a significant association with the development and progression of subclinical atherosclerosis, as assessed by the CACS.

NON-ALCOHOLIC FATTY LIVER DISEASE AND ITS ASSOCIATION WITH RISK FOR DIABETES AND CARDIOVASCULAR DISEASE

Adipose tissue is not simply an accumulation of fat; instead, it 
is an organ that stores excess energy in the form of triglycerides, secretes adipocytokines, and engages in cross-talk with other tissues [30]. Insulin is the hormone that causes excess energy to be stored in adipose tissue and not in other organs. However, in people with insulin resistance, excess energy could be stored in other tissues, such as the liver, abdomen, muscle, and perivascular area. This phenomenon is known as ectopic fat accumulation. NAFLD is a disease characterized by fat accumulation in the liver without a history of alcohol drinking [31]. As the liver is an organ where ectopic fat accumulation occurs, if someone has NAFLD, it means that ectopic fat accumulation has already taken place in that person, indicating that insulin resistance has developed.

NAFLD and diabetes are inextricably linked, as shown by many epidemiological studies [32]. It is known that $70 \%$ to $80 \%$ of patients with diabetes have NAFLD, and diabetic complications are more severe in patients with diabetes and NAFLD. It has also been established that NAFLD patients are more prone to non-alcoholic steatohepatitis and fibrosis if they have diabetes; therefore, preventing and controlling both diseases is a key component of CVD prevention.

In another study, we analyzed the associations of the visceralto-subcutaneous abdominal fat ratio (VSR) with NAFLD and liver fibrosis [33]. In 7,456 KSHS examinees, the presence of fibrosis was defined by the fibrosis-4 score (FIB-4), and a high VSR predicted NAFLD risk and advanced fibrosis as assessed by the FIB-4 score.

We also retrospectively analyzed the association between insulin concentration and NAFLD risk [34]. In 4,956 KSHS subjects, higher baseline insulin concentrations were associated with a higher risk of developing NAFLD after 5 years, suggesting that hyperinsulinemia influences the development of NAFLD. Perhaps unsurprisingly, few studies have measured insulin levels in such a large number of subjects. In another study that analyzed the association between baseline homeostasis model assessment of insulin resistance (HOMA-IR) and NAFLD in 32,060 KSHS participants with 8 years of followup, we found that the degree of baseline insulin resistance, as assessed by HOMA-IR values, was positively associated with future fibrosis progression in patients with NAFLD who did not have diabetes.

The evidence from recent studies suggests that NAFLD is a CVD risk factor [35]. In 21,335 participants in the KSHS, the risk for CAC was compared between those with or without NAFLD and obesity assessed by body mass index. The risk for $\mathrm{CAC}$ was the highest in the group with both abnormalities, and the NAFLD-only group showed a significantly higher risk for CAC than the abdominal obesity-only group, suggesting that the presence of NAFLD could increase the risk for subclinical atherosclerosis regardless of the presence of obesity. In another study performed in 1,575 KSHS participants, the risk for CAC development was the highest in subjects with both NAFLD and high-sensitivity C-reactive protein (hs-CRP) levels $\geq 0.06 \mathrm{mg}$ / $\mathrm{dL}$, followed by those with only NAFLD, those with only hs$\mathrm{CRP} \geq 0.06 \mathrm{mg} / \mathrm{dL}$, and those without NAFLD and hs-CRP $<0.06 \mathrm{mg} / \mathrm{dL}$, suggesting that the concomitant presence of NAFLD and systemic inflammation, as assessed by hs-CRP, increases the risk of CAC development.

NAFLD is one of the hottest research areas in the field of endocrinology and metabolism, and it has recently attracted considerable interest due to the increasing prevalence of metabolic diseases related to obesity, such as diabetes and MS. Further research is warranted to elucidate the relationship of NAFLD with other metabolic diseases.

\section{RISK FACTOR VARIABILITY AND RISK FOR DIABETES AND ATHEROSCLEROSIS}

There has recently been intense interest in the relationship between the variability of CVD risk factors and the risk of actual CVD or diabetes [36]. The details of the associations between the variability of CVD risk factors and increased CVD or diabetes risk vary among individual risk factors; however, a common mechanism could be increased oxidative stress and inflammation caused by the instability of risk factors.

We analyzed the relationship between the variability of total cholesterol (TC) levels and the risk for type 2 diabetes development in 2,827,950 participants of NHIS health check-ups without diabetes [37]. The highest risk of diabetes development was found in the highest decile of cholesterol variation compared with the lowest decile. Consistent results were obtained regardless of the use of anti-hyperlipidemic medication and baseline TC levels, suggesting that participants with a large variation in TC levels showed a higher risk of diabetes development.

In another study, we analyzed the association between body weight variability and the risk of diabetes development [38]. In 4,818 KSHS examinees in whom health examinations were performed in 5 consecutive years, high body weight variability had a significant association with an increased risk of diabetes development over 4 years, and this was particularly evident in those who were overweight or obese.

The hemoglobin glycation index (HGI) is calculated by sub- 
tracting the predicted $\mathrm{HbA} 1 \mathrm{c}$ level from the measured HbAlc level. The predicted $\mathrm{HbA} 1 \mathrm{c}$ level is calculated on the basis of the observed mean blood glucose level by using a linear regression equation between blood glucose and HbA1c levels. Individuals with a high or low HGI have HbA1c levels that are higher or lower than those expected from their blood glucose levels; these could be considered individual signatures $[39,40]$. We analyzed the association between incident CAC and changes in the HGI among participants without diabetes over 4 years [41]. Participants with a high baseline HGI and consistently high HGI showed a higher risk for incident CAC than those with a low baseline HGI, and an increased HGI over 4 years significantly increased the risk for CAC regardless of baseline HbA1c levels.

\section{THE ASSOCIATIONS BETWEEN OBESITY DEGREE, GLYCEMIC STATUS, AND OTHER DISEASES}

Recent Korean nationwide studies have suggested associations between glycemic status, obesity status, and various diseases in large numbers of participants. In 9,720,220 Korean adults who underwent national health examinations in 2009, the risk of HF was assessed according to baseline obesity and glycemic status [42]. Obesity showed an inverse-J-shape correlation with the risk of $\mathrm{HF}$, and the risk increased in people with low body weight and those with class II or higher obesity. In addition, we, for the first time in the literature for a Korean population, reported $8 \%$ and $86 \%$ increased risks for $\mathrm{HF}$ in individuals with prediabetes and diabetes.

Furthermore, we analyzed the association between glycemic status and the risk of Parkinson's disease in 8,443,353 Koreans who underwent national health examinations from 2009 to 2010 and were followed up until 2016 for the assessment of Parkinson's disease [43]. The risk of Parkinson's disease significantly increased as the baseline glycemic status worsened from normoglycemia to diabetes with a longer duration.

Although the exact mechanism underlying the association between these two diseases has not been fully elucidated, mitochondrial dysfunction, endoplasmic reticulum stress, chronic

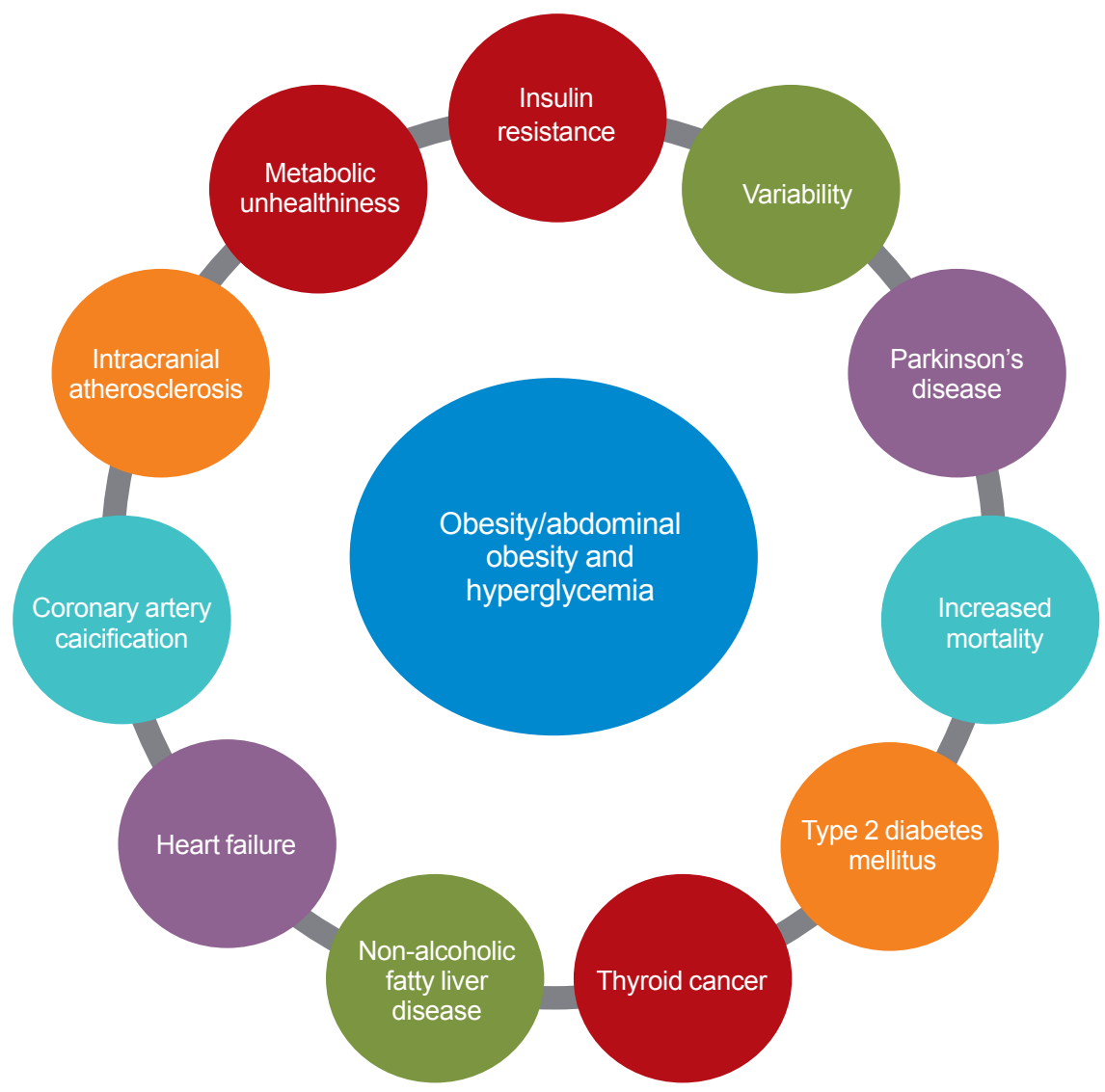

Fig. 1. Obesity and its associations with metabolic diseases and vascular health. 
low-grade inflammation, and alterations in metabolism are thought to cause insulin resistance and ultimately neurodegenerative disorders, as well as diabetes.

\section{CONCLUSIONS}

The prevalence of risk factors related to MS, such as obesity, hyperglycemia, hypertension, and dyslipidemia is rapidly increasing, and variability of these risk factors is known to be related to unfavorable outcomes in terms of CVD and diabetes. NAFLD, which was thought to be only caused by fatty infiltration of the liver, is a well-known cause of diabetes, as well as a consequence of diabetes (and vice versa). Researchers have recently focused on NAFLD as a significant risk factor for CVD. In addition, MHO was a hot area of research due to the unknown mechanism by which obesity might not increase CVD risk, but recently published studies have debated the existence of $\mathrm{MHO}$ and argued that it is highly dependent on the definition. Lastly, as the global population of people with obesity and diabetes has rapidly increased, it has been reported that obesity and diabetes are related to diseases that were previously thought to be unrelated, such as HF and Parkinson's disease.

As the content of the present review was drawn primarily from the results of the author's own research, it might not have covered some of the puzzles that remain to be solved. However, these results allow us to conclude that the phenotypes of obesity can be completely different depending on the depots of accumulated fat, the corresponding muscle amount, and the presence of ectopic fat accumulation. Furthermore, NAFLD is a field of interest for both endocrinologists and hepatologists, and it is an area where excellent results can be achieved through collaboration. As NAFLD is considered to be a risk factor for CVD, further research in this field could prevent CVD in patients with obesity and diabetes. The most important lesson from research in this field is that when it comes to treating obesity, it is important to focus not just on losing weight, but losing weight in a healthy way, so that our patients can live without obesity-related comorbidities as long as possible.

In conclusion, from the results of the author's own research, obesity (especially abdominal obesity) and its variability are strongly associated with various metabolic diseases related to ectopic fat accumulation and insulin resistance. In addition, hyperglycemia and insulin resistance, which could be caused by abdominal obesity, are related to type 2 diabetes and other unexpected diseases, such as Parkinson's disease and HF. Finally, these relationships between metabolic risk factors could all in- fluence vascular health, eventually leading to an increased risk of CVD (Fig. 1).

\section{CONFLICTS OF INTEREST}

No potential conflict of interest relevant to this article was reported.

\section{ACKNOWLEDGMENTS}

I, Eun-Jung Rhee, would like to express my sincere gratitude to emeritus professor Hun Ki Min (Namgok) for giving me the prestigious Namgok Award and hope that he rests in peace. I would like to thank Prof. Sun-Woo Kim, Prof. Won-Young Lee, Prof. Sung Koo Kang, Prof. Moo-Il Kang, Prof. Ki-Hyun Baek, and Prof. Ki-Won Oh for their unlimited support for my career as a researcher and as a clinician. I also would like to thank my colleagues in my department and my excellent fellows who had been trained in our department for their support. Finally, I would like to thank Prof. Mee Kyoung Kim and Hye Yeon Jang, the managing editor of Endocrinology and Metabolism (EnM), for her passion and love for EnM.

\section{ORCID}

Eun-Jung Rhee https://orcid.org/0000-0002-6108-7758

\section{REFERENCES}

1. World Health Organization. Overweight and obesity [Internet]. Geneva: WHO; 2021 [cited 2022 Jan 25]. Available from: https://www.who.int/news-room/fact-sheets/detail/ obesity-and-overweight.

2. Jarolimova J, Tagoni J, Stern TA. Obesity: its epidemiology, comorbidities, and management. Prim Care Companion CNS Disord 2013; 15:PCC.12f01475.

3. Gallagher EJ, LeRoith D. Obesity and diabetes: the increased risk of cancer and cancer-related mortality. Physiol Rev 2015;95:727-48.

4. Nam GE, Kim YH, Han K, Jung JH, Rhee EJ, Lee WY, et al. Obesity fact sheet in Korea, 2020: prevalence of obesity by obesity class from 2009 to 2018. J Obes Metab Syndr 2021;30:141-8.

5. Bhupathiraju SN, Hu FB. Epidemiology of obesity and diabetes and their cardiovascular complications. Circ Res 2016; 118:1723-35. 
6. Lim S. Ectopic fat assessment focusing on cardiometabolic and renal risk. Endocrinol Metab (Seoul) 2014;29:1-4.

7. Bays HE. "Sick fat," metabolic disease, and atherosclerosis. Am J Med 2009;122(1 Suppl):S26-37.

8. Lee YH, Han K, Ko SH, Ko KS, Lee KU; Taskforce Team of Diabetes Fact Sheet of the Korean Diabetes Association. Data analytic process of a nationwide population-based study using national health information database established by National Health Insurance Service. Diabetes Metab J 2016;40:79-82.

9. Jung CH, Son JW, Kang S, Kim WJ, Kim HS, Kim HS, et al. Diabetes fact sheets in Korea, 2020: an appraisal of current status. Diabetes Metab J 2021;45:1-10.

10. Kim HC, Cho SMJ, Lee H, Lee HH, Baek J, Heo JE, et al. Korea hypertension fact sheet 2020: analysis of nationwide population-based data. Clin Hypertens 2021;27:8.

11. Rhee EJ. Prevalence and current management of cardiovascular risk factors in Korean adults based on fact sheets. Endocrinol Metab (Seoul) 2020;35:85-94.

12. Korean Diabetes Association. Diabetes Fact Sheet in Korea [Internet]. Seoul: KDA; 2019 [cited 2022 Jan 25]. Available from: https://www.diabetes.or.kr/pro/news/admin.php?categ ory $=$ A\&code $=$ admin $\&$ mode $=$ view $\&$ number $=1788$.

13. Eckel RH, Grundy SM, Zimmet PZ. The metabolic syndrome. Lancet 2005;365:1415-28.

14. Alberti KG, Eckel RH, Grundy SM, Zimmet PZ, Cleeman JI, Donato KA, et al. Harmonizing the metabolic syndrome: a joint interim statement of the International Diabetes Federation Task Force on Epidemiology and Prevention; National Heart, Lung, and Blood Institute; American Heart Association; World Heart Federation; International Atherosclerosis Society; and International Association for the Study of Obesity. Circulation 2009;120:1640-5.

15. Brown WV. Different definitions of the metabolic syndrome and risk for recurrent cardiovascular disease. Nat Clin Pract Endocrinol Metab 2006;2:492-3.

16. Lee WY, Park JS, Noh SY, Rhee EJ, Kim SW, Zimmet PZ. Prevalence of the metabolic syndrome among 40,698 Korean metropolitan subjects. Diabetes Res Clin Pract 2004;65: 143-9.

17. Kondo T, Osugi S, Shimokata K, Honjo H, Morita Y, Yamashita K, et al. Metabolic syndrome and all-cause mortality, cardiac events, and cardiovascular events: a follow-up study in 25,471 young- and middle-aged Japanese men. Eur J Cardiovasc Prev Rehabil 2011;18:574-80.

18. Ford ES. The metabolic syndrome and mortality from car- diovascular disease and all-causes: findings from the National Health and Nutrition Examination Survey II Mortality Study. Atherosclerosis 2004;173:309-14.

19. Sung KC, Rhee EJ, Ryu S, Kim BJ, Kim BS, Lee WY, et al. Increased cardiovascular mortality in subjects with metabolic syndrome is largely attributable to diabetes and hypertension in 159,971 Korean adults. J Clin Endocrinol Metab 2015; 100:2606-12.

20. Seo MH, Rhee EJ. Metabolic and cardiovascular implications of a metabolically healthy obesity phenotype. Endocrinol Metab (Seoul) 2014;29:427-34.

21. Rumberger JA, Simons DB, Fitzpatrick LA, Sheedy PF, Schwartz RS. Coronary artery calcium area by electron-beam computed tomography and coronary atherosclerotic plaque area. A histopathologic correlative study. Circulation 1995; 92:2157-62.

22. Detrano R, Guerci AD, Carr JJ, Bild DE, Burke G, Folsom $\mathrm{AR}$, et al. Coronary calcium as a predictor of coronary events in four racial or ethnic groups. N Engl J Med 2008;358: 1336-45.

23. Blankstein R, Gupta A, Rana JS, Nasir K. The implication of coronary artery calcium testing for cardiovascular disease prevention and diabetes. Endocrinol Metab (Seoul) 2017;32: 47-57.

24. Rhee EJ, Seo MH, Kim JD, Jeon WS, Park SE, Park CY, et al. Metabolic health is more closely associated with coronary artery calcification than obesity. PLoS One 2013;8: e74564.

25. Rhee EJ, Lee MK, Kim JD, Jeon WS, Bae JC, Park SE, et al. Metabolic health is a more important determinant for diabetes development than simple obesity: a 4-year retrospective longitudinal study. PLoS One 2014;9:e98369.

26. Smith GI, Mittendorfer B, Klein S. Metabolically healthy obesity: facts and fantasies. J Clin Invest 2019;129:3978-89.

27. Aronson D, Rayfield EJ. How hyperglycemia promotes atherosclerosis: molecular mechanisms. Cardiovasc Diabetol 2002;1:1.

28. Rhee EJ, Park SE, Chang Y, Ryu S, Lee WY. Baseline glycemic status and mortality in 241,499 Korean metropolitan subjects: a Kangbuk Samsung Health Study. Metabolism 2016;65:68-77.

29. Rhee EJ, Kim JH, Park HJ, Park SE, Oh HG, Park CY, et al. Increased risk for development of coronary artery calcification in insulin-resistant subjects who developed diabetes: 4-year longitudinal study. Atherosclerosis 2016;245:132-8.

30. Kershaw EE, Flier JS. Adipose tissue as an endocrine organ. 
J Clin Endocrinol Metab 2004;89:2548-56.

31. Adams LA, Anstee QM, Tilg H, Targher G. Non-alcoholic fatty liver disease and its relationship with cardiovascular disease and other extrahepatic diseases. Gut 2017;66:113853.

32. Rhee EJ. Nonalcoholic fatty liver disease and diabetes: an epidemiological perspective. Endocrinol Metab (Seoul) 2019;34:226-33.

33. Jung CH, Rhee EJ, Kwon H, Chang Y, Ryu S, Lee WY. Visceral-to-subcutaneous abdominal fat ratio is associated with nonalcoholic fatty liver disease and liver fibrosis. Endocrinol Metab (Seoul) 2020;35:165-76.

34. Rhee EJ, Lee WY, Cho YK, Kim BI, Sung KC. Hyperinsulinemia and the development of nonalcoholic fatty liver disease in nondiabetic adults. Am J Med 2011;124:69-76.

35. Lee DY, Rhee EJ, Chang Y, Sohn CI, Shin HC, Ryu S, et al. Impact of systemic inflammation on the relationship between insulin resistance and all-cause and cancer-related mortality. Metabolism 2018;81:52-62.

36. Lee SH, Kim MK, Rhee EJ. Effects of cardiovascular risk factor variability on health outcomes. Endocrinol Metab (Seoul) 2020;35:217-26.

37. Rhee EJ, Han K, Ko SH, Ko KS, Lee WY. Increased risk for diabetes development in subjects with large variation in total cholesterol levels in 2,827,950 Koreans: a nationwide popu- lation-based study. PLoS One 2017;12:e0176615.

38. Rhee EJ, Cho JH, Kwon H, Park SE, Park CY, Oh KW, et al. Increased risk of diabetes development in individuals with weight cycling over 4 years: the Kangbuk Samsung Health study. Diabetes Res Clin Pract 2018;139:230-8.

39. Hempe JM, Gomez R, McCarter RJ Jr, Chalew SA. High and low hemoglobin glycation phenotypes in type 1 diabetes: a challenge for interpretation of glycemic control. J Diabetes Complications 2002;16:313-20.

40. McCarter RJ, Hempe JM, Gomez R, Chalew SA. Biological variation in $\mathrm{HbA} 1 \mathrm{c}$ predicts risk of retinopathy and nephropathy in type 1 diabetes. Diabetes Care 2004;27:1259-64.

41. Rhee EJ, Cho JH, Kwon H, Park SE, Park CY, Oh KW, et al. Association between coronary artery calcification and the hemoglobin glycation index: the Kangbuk Samsung Health Study. J Clin Endocrinol Metab 2017;102:4634-41.

42. Rhee EJ, Kwon H, Park SE, Han KD, Park YG, Kim YH, et al. Associations among obesity degree, glycemic status, and risk of heart failure in 9,720,220 Korean adults. Diabetes Metab J 2020;44:592-601.

43. Rhee SY, Han KD, Kwon H, Park SE, Park YG, Kim YH, et al. Association between glycemic status and the risk of Parkinson disease: a nationwide population-based study. Diabetes Care 2020;43:2169-75. 